\title{
Incidência e fatores associados a complicações em feridas operatórias de mulheres mastectomizadas
}

\author{
Incidence and factors associated with complications in surgical wounds of women after mastectomy
}

Incidencia y factores asociados a complicaciones en heridas quirúrgicas de mujeres mastectomizadas

\author{
Isabela da Rosa Noronha' @ ; Isabele da Rosa Noronha' @ ; Carolina Siqueira Dantas' ${ }^{\circledR}$; \\ Lucia Helena Garcia Penna" ㄹ; Rafael Tavares Jomar' ำ \\ IInstituto Nacional de Câncer, Rio de Janeiro, RJ, Brasil; "Universidade do Estado do Rio de Janeiro, Rio de Janeiro, RJ, Brasil
}

\begin{abstract}
RESUMO
Objetivo: descrever a incidência de complicações em feridas operatórias de mastectomia e identificar fatores associados. Método: estudo retrospectivo desenvolvido em uma coorte hospitalar de 545 mulheres mastectomizadas por câncer de mama no ano 2018 em um centro de assistência de alta complexidade em oncologia da cidade do Rio de Janeiro, Brasil, após aprovação por Comitê de Ética em Pesquisa. Os dados foram coletados a partir dos prontuários, permitindo cálculos da taxa de incidência e da razão da taxa de incidência para cada complicação. Resultados: a complicação que apresentou maior taxa de incidência foi o sangramento (57,14/100 mastectomias-dia), tendo como fatores associados a raça/cor da pele não branca (Razão da Taxa de Incidência: 3,11$)$ e a diabetes mellitus (Razão da Taxa de Incidência: 0,48). Conclusão: os fatores associados ao sangramento da ferida operatória apontam para a necessidade de novas práticas no cuidado ao pós-operatório de mulheres mastectomizadas.

Descritores: Oncologia; Enfermagem; Mastectomia; Ferida Cirúrgica; Fatores de Risco.
\end{abstract}

\begin{abstract}
Objective: to describe the incidence of complications in mastectomy surgical wounds and to identify associated factors. Method: this retrospective study was conducted in a hospital cohort of 545 women mastectomized for breast cancer in 2018 at a high-complexity cancer care center Rio de Janeiro City, Brazil, after approval by the research ethics committee. Data were collected from medical records, allowing incidence rate and incidence rate ratio to be calculated for each complication. Results: the complication with the highest incidence rate was bleeding (57.14/100 mastectomies-day), associated factors being nonwhite race/skin color (incidence rate ratio 3.11) and diabetes mellitus (incidence rate ratio 0.48). Conclusion: the factors associated with bleeding from the surgical wound point to the need for new practices in post-operative care for women with mastectomies.

Descriptors: Medical Oncology; Nursing; Mastectomy; Surgical Wound; Risk Factors.

\section{RESUMEN}

Objetivo: describir la incidencia de complicaciones en heridas quirúrgicas de mastectomía e identificar factores asociados. Método: estudio retrospectivo desarrollado en una cohorte hospitalaria de 545 mujeres mastectomizadas por cáncer de mama en 2018 en un centro de atención de alta complejidad en oncología de la ciudad de Río de Janeiro, Brasil, previa aprobación del Comité de Ética en Investigación. Los datos se obtuvieron de las historias clínicas, lo que permitió calcular la tasa de incidencia y el cociente de la tasa de incidencia para cada complicación. Resultados: la complicación que presentó mayor tasa de incidencia fue el sangrado (57,14 / 100 mastectomías-día), con factores asociados a la raza / color de piel no blanca (índice de tasa de incidencia: 3,11 ) y diabetes mellitus (índice de tasa de incidencia: 0,48 ). Conclusión: los factores asociados al sangrado de la herida quirúrgica apuntan a la necesidad de nuevas prácticas en el cuidado al postoperatorio de las mujeres con mastectomía. Descriptores: Oncología Médica; Enfermería; Mastectomía; Herida Quirúrgica; Factores de Riesgo.
\end{abstract}

\section{INTRODUÇÃO}

O objeto deste estudo são as complicações de ferida operatória de mastectomia e seus fatores associados. 0 câncer de mama é uma doença heterogênea, que pode ser dividida em diversos subtipos clínicos e histológicos ${ }^{1}$. Atualmente, é um dos principais problemas de saúde pública. Ao excluir o câncer de pele não melanoma, é o tipo de câncer que mais acomete as mulheres no Brasil e no mundo e sua incidência vem aumentando gradativamente².

Estima-se que no triênio 2020-2022 tenham 66.280 casos novos de câncer de mama para cada ano. Este é o câncer mais incidente em mulheres da região Sul, Sudeste, Centro Oeste e Nordeste e Norte do Brasil ${ }^{1}$.

Entre os tratamentos ofertados para o câncer de mama destacam-se a quimioterapia, a radioterapia e a cirurgia. O tratamento cirúrgico pode ser classificado em: 1) Terapia conservadora de mama, que realiza exérese do tumor preservando o máximo de tecido possível, como a quadrantectomia e segmentectomia;2) Mastectomia, que é a forma de tratamento mais eficaz, embora tenha um caráter mutilador, podendo ser dividida em seis tipos: mastectomia simples, dupla ou bilateral, poupadora de pele, poupadora de mamilo, radical modificada e radical ${ }^{3}$. 
As cirurgias de mama podem resultar em complicações de ferida operatória, como: infecção $0^{4-9}$, necrose $e^{4,6,10}$ seroma $^{4,6,7,9,10}$, deiscência ${ }^{4,9}$, sangramento ${ }^{8,9}$, hematoma ${ }^{4,6,7,10}$ e epidermólise ${ }^{11}$. Tais complicações repercutem diretamente na vida desta mulher, pois pode gerar reinternações, aumentar os gastos hospitalares, mobilizar toda equipe de saúde, provocar problemas psicossociais e incapacitantes, podendo inclusive levar o paciente a óbito ${ }^{12}$ ou ao atraso do início da terapia adjuvante ${ }^{13}$.

O intuito em estudar as complicações em feridas operatórias de mastectomia visa qualificar a assistência de enfermagem para esta clientela, com enfoque em melhores intervenções pela estomaterapia, a fim de proporcionar melhor qualidade de vida para as usuárias. Acrescenta-se a essa relevância em se estudar a temática a epidemiologia da doença, sendo a mais incidente no mundo entre as mulheres com taxa de incidência estimada para o Brasil de 61,61 casos novos para cada 100 mil mulheres ${ }^{1}$.

Dessa forma, os objetivos deste estudo são descrever a incidência de complicações nas feridas operatórias de mastectomia e identificar fatores associados.

\section{REVISÃO DE LITERATURA}

As complicações das feridas operatórias de mastectomia podem ser divididas em três fases, sendo estas: imediata, que ocorre em até 24 horas após a cirurgia; mediata, quando surge até sete dias de pós operatório; tardia quando acontece após retirada do dreno e pontos e a paciente tem alta hospitalar ${ }^{14}$.

Dentre os impactos decorrentes do tratamento cirúrgico, destaca-se como principal fator negativo, a sensação de vergonha associada ao distúrbio de imagem corporal, influenciando na qualidade de vida e na saúde dessas mulheres ${ }^{15,16}$. Contudo, o sofrimento diante da perda da mama permeia diversos aspectos, como a baixa estima, não se sentir mulher, ausência de significado na vida, abandono ou redução das atividades na rotina diária e trabalho devido às limitações da mastectomia e sensação de inutilidade ${ }^{17}$.

Alguns fatores que interferem no processo de cicatrização da ferida operatória são descritos na literatura. Dentre estes, destacam-se: infecção local, deficiência da técnica cirúrgica, tensão excessiva das bordas da ferida, baixa perfusão nesta área, processo traumático na ferida no pós-operatório, radioterapia e cirurgia de emergência. Com relação aos fatores sistêmicos, mencionam-se idade, desnutrição, obesidade, tabagismo, comprometimento imunológico, uso crônico de medicamentos esteroidais ou imunoterápicos ${ }^{18}$. Desse modo, a determinação dos fatores que afetam o processo de cicatrização das feridas operatórias é fundamental para a realização de medidas de prevenção de complicações e tratamento das mesmas.

\section{MÉTOdo}

Trata-se de um estudo de coorte retrospectiva que teve como população alvo mulheres submetidas à mastectomia por câncer de mama no ano 2018 em um centro de assistência de alta complexidade em oncologia localizado na cidade do Rio de Janeiro, Brasil. Aquelas submetidas à reconstrução mamária foram consideradas inelegíveis para participação no estudo, por não serem acompanhadas pela equipe de enfermagem ambulatoriamente no pós-operatório.

A fim de identificar complicações nas feridas operatórias, acompanhou-se a evolução das pacientes durante 60 dias após a realização da mastectomia por meio da avaliação de seus prontuários. Assim, foram selecionados 545 prontuários de mulheres que realizaram mastectomia. O tempo de seguimento aqui eleito baseou-se em um estudo $^{19}$, que identificou a ocorrência de complicações em ferida cirúrgica até 60 dias após a realização de mastectomia.

Para conhecer o perfil das mulheres mastectomizadas, foram coletadas dos prontuários delas as seguintes informações: idade (< 60 ou $\geq 60$ anos), cor da pele (branca ou não branca), tabagismo (tabagista/ex-tabagista ou não tabagista), índice de massa corporal ${ }^{20}\left(\leq 18,4 \mathrm{~kg} / \mathrm{m}^{2}\right.$ : baixo; $18,5-24,9 \mathrm{~kg} / \mathrm{m}^{2}$ : adequado; $25,0-29,9 \mathrm{~kg} / \mathrm{m}^{2}$ : sobrepeso; $\geq$ $30 \mathrm{~kg} / \mathrm{m}^{2}$ : obesidade), hipertensão arterial (sim ou não), diabetes mellitus (sim ou não), cirurgia mamária prévia (sim ou não) e quimioterapia neoadjuvante (sim ou não). Também foram coletadas informações sobre o tipo de mastectomia (simples ou radical modificada) e as complicações ocorridas durante o seguimento (infecção, celulite, sangramento, hematoma, necrose, deiscência, epidermólise ou seroma).

A fim de avaliar a frequência de complicação em ferida cirúrgica estimou-se a taxa de incidência de cada tipo de complicação. Para tanto, dividiu-se o número de cada tipo de complicação ocorrida no período do estudo pelo somatório dos tempos individuais de seguimento (em dias), isto é, a quantidade de pessoa-tempo. Em seguida, o resultado dessa divisão foi multiplicado por 100 e a taxa de incidência foi apresentada por 100 mastectomias-dia. 
Calcularam-se a taxa de incidência para cada complicação e a razão da taxa de incidência (RTI) acompanhada dos respectivos intervalos de $95 \%$ de confiança (IC95\%) segundo variáveis de exposição (idade, cor da pele, tabagismo, índice de massa corporal, hipertensão arterial, diabetes mellitus, cirurgia mamária prévia, quimioterapia neoadjuvante e tipo de mastectomia). A RTI é uma medida de associação que avalia quantas vezes a taxa de incidência é maior (ou menor) no grupo de expostos em comparação ao grupo de não expostos ${ }^{21}$. Para a complicação infecção, considerou-se até 30 dias de pós-operatório para calcular a taxa de incidência e a RTI ${ }^{22}$.

Cabe destacar que as medidas de frequência (taxa de incidência) e de associação (RTI) aqui calculadas são as mais apropriadas para populações dinâmicas ${ }^{21}$, tal qual a população do presente estudo, uma vez que as mulheres entraram na coorte e saíram dela em diferentes momentos do seguimento.

Em obediência às diretrizes e normas regulamentadoras de pesquisas envolvendo seres humanos no Brasil, constantes na Resolução no 466, de 12 de dezembro de 2012, do Conselho Nacional de Saúde do Ministério da Saúde, a condução deste estudo foi aprovada pelo Comitê de Ética em Pesquisa da instituição.

\section{RESULTADOS}

Com relação as características demográficas e clínicas das participantes, a maior parte é composta por mulheres com menos de 60 anos de idade (53,76\%), sendo a idade mínima 23 e máxima 86 anos, de raça/cor de pele não branca $(63,17 \%)$, não tabagista $(65,67 \%)$, obesa $(37,06 \%)$, hipertensa $(52,39 \%)$, não diabética $(79,48 \%)$ e que não realizou cirurgia mamária prévia $(94,00 \%)$. Com relação ao tratamento, a maioria realizou tratamento neoadjuvante $(70,46 \%)$ e mastectomia radical modificada (62,39\%).

A Tabela 1 mostra a frequência de cada complicação e a respectiva taxa de incidência. A complicação que apresentou maior taxa de incidência foi o sangramento (57,14/100 mastectomias-dia), seguido do hematoma $(12,18 / 100$ mastectomias-dia) e da epidermólise (10,92/100 mastectomias-dia).

TABELA 1: Taxa de incidência de cada tipo de complicação em ferida cirúrgica de um Centro de assistência de alta complexidade em oncologia. Rio de Janeiro, RJ, Brasil, 2020

\begin{tabular}{lcc}
\hline Tipo de complicação & $\mathbf{N}$ & Taxa de incidência por 100 mastectomias-dia \\
\hline Sangramento & 20 & 57,14 \\
Hematoma & 39 & 12,18 \\
Epidermólise & 100 & 10,92 \\
Celulite & 02 & 7,40 \\
Infecção* & 47 & 6,40 \\
Seroma & 380 & 5,72 \\
Necrose & 151 & 5,46 \\
Deiscência & 140 & 4,21 \\
\hline
\end{tabular}

*Incidência em até 30 dias

No tocante ao sangramento, única complicação que apresentou associação com as variáveis de exposição aqui estudadas (Tabelas 2 e 3), observaram-se maiores taxas de incidência entre as mulheres não brancas $(\mathrm{RTI}=3,11$; IC95\%:1,12-9,87) em comparação às brancas e menores taxas de incidência entre as que tinham diabetes mellitus quando comparadas àquelas que não apresentavam diabetes mellitus (RTI=0,48; IC95\%: 0,17$1,53)$. 
TABELA 2: Razão de taxa de incidência (RTI) de sangramento, hematoma, epidermólise, celulite e respectivo intervalo de $95 \%$ de confiança (IC95\%) de um Centro de assistência de alta complexidade em oncologia. Rio de Janeiro, RJ, Brasil, 2020.

\begin{tabular}{|c|c|c|c|c|c|}
\hline Variáveis & & $\begin{array}{c}\text { Sangramento } \\
\text { RTI (IC95\%) }\end{array}$ & $\begin{array}{l}\text { Hematoma } \\
\text { RTI (IC95\%) }\end{array}$ & $\begin{array}{c}\text { Epidermólise } \\
\text { RTI (IC95\%) }\end{array}$ & $\begin{array}{c}\text { Seroma } \\
\text { RTI (IC95\%) }\end{array}$ \\
\hline \multirow[t]{2}{*}{ Idade } & $<60$ anos & 1,00 & 1,00 & 1,00 & 1,00 \\
\hline & $\geq 60$ anos & $0,40(0,14-1,07)$ & $0,87(0,43-1,86)$ & $1,04(0,69-1,58)$ & $0,96(0,78-1,18)$ \\
\hline \multirow[t]{2}{*}{ Raça/cor da pele } & Branca & 1,00 & 1,00 & 1,00 & 1,00 \\
\hline & Não branca & $3,11(1,12-9,87)$ & $1,28(0,62-2,72)$ & $1,09(0,70-1,70)$ & $0,97(0,78-1,21)$ \\
\hline \multirow[t]{2}{*}{ Tabagismo } & Não tabagista & 1,00 & 1,00 & 1,00 & 1,00 \\
\hline & Tabagista/ex-tabagista & $0,48(0,18-1,33)$ & $1,02(0,50-2,03)$ & $1,05(0,69-1,59)$ & $0,96(0,77-1,20)$ \\
\hline \multirow{2}{*}{$\begin{array}{l}\text { Índice de massa } \\
\text { corporal }\end{array}$} & Baixo/adequado & 1,00 & 1,00 & 1,00 & 1,00 \\
\hline & Sobrepeso/Obesidade & - & - & - & - \\
\hline \multirow{2}{*}{$\begin{array}{l}\text { Hipertensão } \\
\text { arterial }\end{array}$} & Não & 1,00 & 1,00 & 1,00 & 1,00 \\
\hline & Sim & $0,48(0,17-1,53)$ & $1,44(0,72-2,94)$ & $1,07(0,70-1,63)$ & $0,96(0,78-1,19)$ \\
\hline \multirow[t]{2}{*}{ Diabetes mellitus } & Não & 1,00 & 1,00 & 1,00 & 1,00 \\
\hline & Sim & $0,31(0,08-0,91)$ & $0,95(0,45-1,93)$ & $0,99(0,53-1,61)$ & $1,01(0,77-1,28)$ \\
\hline \multirow{2}{*}{$\begin{array}{l}\text { Cirurgia mamária } \\
\text { anterior }\end{array}$} & Não & 1,00 & 1,00 & 1,00 & 1,00 \\
\hline & $\operatorname{Sim}$ & $1,78(0,43-11,25)$ & $0,79(0,92-3,10)$ & $1,64(0,33-4,95)$ & $1,05(0,67-1,57)$ \\
\hline \multirow{2}{*}{$\begin{array}{l}\text { Quimioterapia } \\
\text { neoadjuvante }\end{array}$} & Não & 1,00 & 1,00 & 1,00 & 1,00 \\
\hline & Sim & $2,18(0,81-5,84)$ & $0,94(0,45-2,05)$ & $0,95(0,61-1,51)$ & $1,01(0,80-1,27)$ \\
\hline \multirow{2}{*}{$\begin{array}{l}\text { Tipo de } \\
\text { mastectomia }\end{array}$} & Simples & 1,00 & 1,00 & 1,00 & 1,00 \\
\hline & Radical modificada & - & - & - & - \\
\hline
\end{tabular}

TABELA 3: Razão de taxa de incidência (RTI) de infecção, seroma, necrose, deiscência e respectivo intervalo de $95 \%$ de confiança (IC95\%) de um Centro de assistência de alta complexidade em oncologia. Rio de Janeiro, RJ, Brasil, 2020.

\begin{tabular}{|c|c|c|c|c|}
\hline Variáveis & $\begin{array}{c}\text { Infecção } \\
\text { RTI (IC95\%) }\end{array}$ & $\begin{array}{c}\text { Seroma } \\
\text { RTI (IC95\%) }\end{array}$ & $\begin{array}{c}\text { Necrose } \\
\text { RTI (IC95\%) }\end{array}$ & $\begin{array}{l}\text { Deiscência } \\
\text { RTI (IC95\%) }\end{array}$ \\
\hline \multicolumn{5}{|l|}{ Idade } \\
\hline$<60$ anos & 1,00 & 1,00 & 1,00 & 1,00 \\
\hline$\geq 60$ anos & $1,01(0,52-1,90)$ & $0,96(0,78-1,18)$ & $0,95(0,68-1,33)$ & $0,94(0,66-1,33)$ \\
\hline \multicolumn{5}{|l|}{ Raça/cor da pele } \\
\hline Branca & 1,00 & 1,00 & 1,00 & 1,00 \\
\hline Não branca & $0,77(0,38-1,70)$ & $0,97(0,78-1,21)$ & $1,01(0,69-1,45)$ & $1,15(0,79-1,69)$ \\
\hline \multicolumn{5}{|l|}{ Tabagismo } \\
\hline Não tabagista & 1,00 & 1,00 & 1,00 & 1,00 \\
\hline Tabagista/ex-tabagista & $0,97(0,52-1,80)$ & $0,96(0,77-1,20)$ & $1,08(0,77-1,51)$ & $1,08(0,75-1,53)$ \\
\hline \multicolumn{5}{|l|}{ Índice de massa corporal } \\
\hline Baixo/adequado & 1,00 & 1,00 & 1,00 & 1,00 \\
\hline Sobrepeso/Obesidade & - & - & - & - \\
\hline \multicolumn{5}{|l|}{ Hipertensão arterial } \\
\hline Não & 1,00 & 1,00 & 1,00 & 1,00 \\
\hline Sim & $0,88(0,47-1,68)$ & $0,96(0,78-1,19)$ & $1,03(0,73-1,47)$ & $0,90(0,64-1,28)$ \\
\hline \multicolumn{5}{|l|}{ Diabetes mellitus } \\
\hline Não & 1,00 & 1,00 & 1,00 & 1,00 \\
\hline Sim & $1,08(0,54-2,06)$ & $1,01(0,77-1,28)$ & $0,98(0,66-1,41)$ & $0,98(0,65-1,43)$ \\
\hline \multicolumn{5}{|l|}{ Cirurgia mamária anterior } \\
\hline Não & 1,00 & 1,00 & 1,00 & 1,00 \\
\hline Sim & $0,86(0,02-5,07)$ & $1,05(0,67-1,57)$ & $1,17(0,52-2,29)$ & $1,17(0,31-3,08)$ \\
\hline \multicolumn{5}{|c|}{ Quimioterapia neoadjuvante } \\
\hline Não & 1,00 & 1,00 & 1,00 & 1,00 \\
\hline Sim & $1,08(0,56-2,20)$ & $1,01(0,80-1,27)$ & $0,99(0,69-1,44)$ & $1,05(0,72-1,57)$ \\
\hline \multicolumn{5}{|l|}{ Tipo de mastectomia } \\
\hline Simples & 1,00 & 1,00 & 1,00 & 1,00 \\
\hline Radical modificada & - & - & - & - \\
\hline
\end{tabular}




\section{DISCUSSÃO}

O objetivo deste estudo foi descrever a incidência de complicações apresentadas nas feridas operatórias de mastectomia e identificar fatores associados. Dentre as complicações investigadas, a única que mostrou associação com as variáveis de exposição foi o sangramento, tendo como fator de risco a raça/cor da pele não branca e como fator protetor a presença de diabetes mellitus.

O sangramento é uma complicação hemorrágica que, em muitos casos, resulta em reabordagem cirúrgica, prolongando o tempo de internação em 1,3 dias e aumentando os custos hospitalares em \$ 5.495 dólares por admissão ${ }^{23}$. Nos limites da comparação, os resultados do presente estudo evidenciam que a incidência de sangramento em cirurgia mamária é superior à observada em estudos realizados nos Estados Unidos $2,54 \%{ }^{23}$ e $12 \%{ }^{24}$. Dentre os principais fatores de risco, destacam-se: obesidade, insuficiência cardíaca congestiva e doença pulmonar crônica ${ }^{23}$. De modo controverso à análise dos dados, outros estudos identificaram diabetes como um fator preditor de sangramento ${ }^{23,25}$. Além disso, não foram encontrados estudos que relacionem o sangramento com a raça/cor da pele.

$\mathrm{Na}$ literatura, observa-se que as principais complicações apresentadas pós-mastectomia são infecção ${ }^{4,9,26}$, hematoma $^{4,27}$, seroma ${ }^{4,5,9,27-29}$, deiscência ${ }^{4,9,27}$, necrose ${ }^{4,27}$, sangramento ${ }^{9}$ e celulite $^{27}$. O que corrobora com o estudo, o qual também inclui a epidermólise como complicação incidente no pós-operatório de mastectomia.

Embora a epidermólise não tenha apresentado associação significativa, convém ressaltar que em estudo realizado, essa complicação ocorreu em $28 \%$ dos pacientes que realizaram cirurgia restauradora na mama ${ }^{11}$. A mesma está associada à técnica cirúrgica, onde uma tensão na linha de sutura causa um sofrimento da pele nessa região, geralmente encontrada em abdominoplastias com incidência entre $5,8^{30}$ a $12,8 \%^{31}$. Outros autores não identificaram a epidermólise como complicação de mastectomia possivelmente devido ao desconhecimento e à facilidade no tratamento com coberturas, sendo necessária a realização de mais estudos para elucidação.

Com relação ao seroma, o mesmo é definido como um acúmulo de líquido seroso localizado entre a parede torácica e a pele, ocorrendo em mais de $50 \%$ das cirurgias da mama ${ }^{28}$. Embora o seroma não tenha se mostrado associado à nenhuma das variáveis de exposição investigadas, estudos mostraram sua ocorrência em cirurgias de mastectomias relacionado ao $\mathrm{IMC}^{29}$, obesidade ${ }^{32}$, técnica de dissecção ${ }^{29}$ e duração total da drenagem da ferida cirúrgica ${ }^{29}$. Já em cirurgias com reconstrução mamária, está associado à sutura ${ }^{33}$ e à hipertensão arterial ${ }^{34}$. Embora seja uma complicação com alta incidência, apresenta baixa necessidade de nova intervenção cirúrgica ${ }^{33}$.

Embora o presente estudo não tenha identificado a associação entre as variáveis de exposição e a incidência de deiscência de ferida operatória, a mesma apresentou taxa de incidência de 4,21/100 mastectomias-dia o que corrobora com estudos, que apresentaram incidência de 2,1\% em mastectomia ${ }^{35}$ e entre $7,2 \%$ a $10 \%$ em mastectomias com reconstrução ${ }^{10,35}$. Entre os fatores de risco para ocorrência de deiscência destacam-se infecção local ${ }^{18}$, estresse excessivo nas bordas da ferida ${ }^{18}$, hipoperfusão da área ${ }^{18}$, radioterapia neoadjuvante $^{36}$, idade avançada ${ }^{18}$ e realização da cirurgia de emergência ${ }^{18}$.

A infecção de sítio cirúrgico caracterizada como complicação apresentada nos primeiros 30 dias após o procedimento cirúrgico, é uma complicação que merece destaque, visto que ocupa o 3o lugar entre as Infecções Relacionadas à Assistência à Saúde e representa 14\% das infecções apresentadas em pacientes hospitalizados, aumentando a mortalidade e o tempo de internação ${ }^{22}$. A análise mostrou taxa de incidência de 6,40/100 mastectomiasdia de infecção de sítio cirúrgico, o que corrobora com estudos que apresentam ocorrência entre 2,05 a 16,2\% 4,8,26,37. A análise dos dados não apresentou associação significativa para esta complicação, entretanto estudos mostraram como principais fatores de risco: presença de obesidade ${ }^{4,8,32}$, diabetes ${ }^{4,25}$, hipertensão ${ }^{4}$, tabagismo ${ }^{4}$, seroma no pósoperatório $^{26}$, hematoma ${ }^{26}$, uso de dispositivo de drenagem no pós-operatório por tempo prolongado ${ }^{26}$, conexão e desconexão de tubos proximais sem práticas assépticas padronizadas ${ }^{26}$. Com relação aos fatores operatórios, destacamse a realização de mastectomia bilateral e reconstrução imediata ${ }^{4}$. Desse modo, é recomendada a remoção do dreno quando o volume de drenagem for inferior a $30-50 \mathrm{ml} /$ dia ou em intervalo de tempo de 7 a 10 dias $^{28}$.

\section{Limitações do estudo}

Dentre as limitações do estudo, destaca-se a possibilidade de ocorrência de viés de classificação das variáveis raça/cor da pele e presença de complicação em ferida operatória, uma vez que se trata de um estudo com uso de dados secundários, registrados por diferentes profissionais. Ademais, a ausência de coleta de dados intraoperatórios (a exemplo do tempo de cirurgia) limitou a análise dos dados. Não obstante as suas limitações, cabe ressaltar a originalidade do estudo, uma vez que os dados foram coletados de uma grande amostra, utilizando uma medida de associação pouco utilizada em estudos de enfermagem, mas apropriada para a população do estudo. Por fim, sugerese que novos estudos sejam realizados incluindo a análise de características intraoperatórios para avaliar a associação desses fatores com a incidência de complicações pós-operatórias. 


\section{CONCLUSÃO}

No centro de assistência de alta complexidade em oncologia investigado, das 545 mastectomias avaliadas em 2018, ocorreram 879 complicações, sendo as mais incidentes o sangramento, o hematoma e a epidermólise. A raça/cor da pele não branca mostrou-se um fator de risco para a incidência de sangramento em ferida operatória de mastectomia e a presença de diabetes mellitus um fator protetor. Assim, o estudo contribui para reflexão sobre a necessidade de novas práticas no cuidado ao pós-operatório de mulheres mastectomizadas.

\section{REFERÊNCIAS}

1. Ministério da Saúde (Br). Instituto Nacional de Câncer. Estimativa 2020-2022: Estimativa de Câncer no Brasil. Brasília (DF): Ministério da Saúde; 2020.

2. Markovic A, Pessoa SGP. Analysis of the participation of a university hospital in a national program for breast reconstruction. Rev. bras. cir. plást. [Internet], 2018 [cited 2019 Nov 18]; 33(3):305-11. DOI: http://www.dx.doi.org/10.5935/21771235.2018RBCP0142

3. Pereira APVM, Santos GRF, Furtado LFT, Molina MA, Luz TFN, Esteves APVS. Impacts of mastectomy and mammoplasty on the life of women with breast câncer. Cad. Saúde Pública [Internet], 2019 [cited 2020 Jan 20]; 2(1):38-52. Available from: https://www.unifeso.edu.br/revista/index.php/cadernosdemedicinaunifeso/article/view/1294/575.

4. Olsen MA, Nickel KB, Margenthaler JA, Fox IK, Ball KE, Mines D, et al. Development of a Risk Prediction Model to Individualize Risk Factors for Surgical Site Infection After Mastectomy. Ann Surg Oncol [Internet], 2016 [cited 2019 Dec 15]; 23(8):2471-9. DOI: https://doi.org/10.1245/s10434-015-5083-1

5. Olasehinde O, Alatise O, Arowolo O, Adisa A, Wuraola F, Boutin-Foster C, et al. Safety and feasibility of early postmastectomy discharge and home drain care in a low resource setting. Jornal of Surgical Oncology [Internet], 2018 [cited 2020 Jan 25]; 118(6):861-6. DOI: https://doi.org/10.1002/jso.25215.

6. Beugels J, Meijvogel JLW, Tuinder SMH, Tjan-Heijnen VCG, Huts EM, Piatkiwski A, et al. The influence of neoadjuvant chemotherapy on complications of immediate DIEP flap breast reconstructions. Breast cancer res treat [Internet], 2019 [cited 2019 Nov 18]; 176(2):367-75. DOI: https://doi.org/10.1007/s10549-019-05241-9.

7. Bear HD, Tang G, Rastogi P, Geyer CR, Zoon CK, Kidwell KM, et al. The Effect on Surgical Complications of Bevacizumab Added to Neoadjuvant Chemotherapy for Breast Cancer: NRG Oncology/NSABP Protocol B-40. Ann Surg Oncol [Internet], 2017 [cited 2019 Nov 25]; 24(7):1853-60. DOI: https://doi.org/10.1245/s10434-016-5662-9.

8. Garland M, Hsu FC, Clark C, Chiba A, Mcnatt MH. The impact of obesity on outcomes for patients undergoing mastectomy using the ACS-NSQIP data set. Breast cancer res treat [Internet], 2018 [cited 2020 Jan 12]; 168(3):723-6. DOI: https://doi.org/10.1007/s10549-017-4651-4.

9. Lee CM, Tan VK, Tan BK, Madhukumar P, Yong WS, Wong CY, et al. Perioperative Outcomes of Therapeutic Breast Surgery in the Elderly. Ann Acad Med Singap [Internet], 2016 [cited 2020 Jan 22]; 45(6):261-3. DOI: https://doi.org/10.1016/S09609776(15)70375-9.

10. El Troudi MAT, Duquea LV, Ortiz JDD, Anguloa B, Portillaa J, Daza DJR. Radical mastectomy with reconstruction immediate Instituto Autónomo Hospital Universitario de Los Andes in the period June 2012-April 2015. Rev Chil Cir [Internet], 2017 [cited 2020 Jan 10]; 69(3):234-46. DOI: http://dx.doi.org/10.1016/j.rchic.2016.11.012.

11. Tavares-Filho JM, Franco D, Moreto L, Porchat C, Franco T. Use of the myocutaneous latissimus dorsi flap with fat extension in breast reconstruction: an option for filling the upper pole. Rev. Bras. Cir. Plást. [Internet], 2015 [cited 2020 Mar 24]; 30(3):1-5. DOI: http://www.dx.doi.org/10.5935/2177-1235.2015RBCP0174.

12. Marques GS, Almeida PF, Farias LRC, Nascimento DC. Preliminary study on operatory wound dehiscence records in a university hospital. Rev. HUPE [Internet], 2017 [cited 2020 Mar 22]; 15(4):312-9. DOI: https://doi.org/10.12957/rhupe.2016.31605.

13. Valente SA, LIU Y, Upadhyaya S, Tu C, Pratt DA. The effect of wound complications following mastectomy with immediate reconstruction on breast cancer recurrence. Am. J. Surg. [Internet], 2019 [cited 2020 Mar 28]; 217(3):514-8. DOI: https://doi.org/10.1016/j.amjsurg.2018.10.028.

14. Marques AA, Silva MP, Amaral TP. Tratado de Fisioterapia em Saúde da Mulher, 1. ed. São Paulo: Roca LTDA; 2011.

15. Oliveira FBM, Silva FS, Prazeres ASB. Impact of breast cancer and mastectomy in female sexuality. Rev. Enferm. UFPE on line. [Internet], 2017 [cited 2020 Jan 10]; 11(6):2533-40. Available from: https://periodicos.ufpe.br/revistas/revistaenfermagem/article/view/23421

16. Nascimento KTS, Fonseca LCT, Andrade SSC, Leite KNS, Costa TF, Oliveira SHS. Feelings and sources of emotional support for women in pre-operative mastectomy in a teaching hospital. Rev. enferm. UERJ [Internet], 2015 [cited 2020 Sep 27]; 23(1):10814. DOI: http://dx.doi.org/10.12957/reuerj.2015.15598.

17. Lago EA, Andrade NKS, Nery IS, Avelino FVSD. Feelings of mastectomy women about self image and changes in daily life. Ciência\&Saúde [Internet], 2015 [cited 2019 Oct 15]; 8(1):15-8. DOI: https://doi.org/10.15448/1983-652X.2015.1.18648.

18. Spira JAO, Borges EL, Silva PAB, Abreu MNS, Guedes ACM, Junior JFP. Factors associated with complex surgical wounds in breast and abdomen: a case-control observational study. Rev. Latinoam. Enferm. [Internet], 2018 [cited 2020 Feb 15]; $26(1)$ :e3052. DOI: https://doi.org/10.1590/1518-8345.2274.3052.

19. Nickel KB, Fox IK, Margenthaler JA, Wallace AE, Fraser VJ, Olsen MA. Effect of Noninfectious Wound Complications after Mastectomy on Subsequent Surgical Procedures and Early Implant Loss. J. Am. Coll. Surg. [Internet], 2016 [cited 2020 Mar 24]; 222(5):844-52. DOI: https://doi.org/10.1016/j.jamcollsurg.2016.01.050. 
20. World Health Organization (WHO). Obesity: preventing and managing the global epidemic: report from a WHO consultation. Geneva: WHO; 2000 [cited 2020 Mar 24]. Available from: http://www.who.int/nutrition/publications/obesity/WHO_TRS_894/en/.

21. Oliveira PFF. Epidemiologia e bioestatística: fundamentos para a leitura crítica. Rio de Janeiro: Rubio; 2015.

22. Agência Nacional de Vigilância Sanitária (ANVISA). Critérios Diagnósticos de Infecção Relacionada à Assistência à Saúde. Brasília (DF): Anvisa; 2017.

23. Nwaogu I, Bommarito K, Olsen MA, Margenthaler JA. Economic impact of bleeding complications after mastectomy. J Surg Res [Internet], 2015 [cited 2020 Jul 18]; 199(1):77-83. DOI: https://doi.org/10.1016/j.jss.2015.03.084.

24. Orr JP, Shammas RL, Thomas AB, Truong T, Cho EH, Kuchibhatla M, et al. Bleeding After Free Flap-Based Breast Reconstruction: A NSQIP Analysis. J. Reconstr. Microsurg. [Internet], 2019 [cited 2020 Aug 15]; 35(6):417-24. DOI: https://doi.org/10.1055/s0038-1677037.

25. Rifkin WJ, Kantar RS, Cammarata MJ, Wilson SC, Diaz-Siso R, Golas AR, et al. Impact of Diabetes on 30-Day Complications in Mastectomy and Implant-Based Breast Reconstruction. J. Surg. Res. [Internet], 2019 [cited 2020 Aug 18]; 235(1):148-59. DOI: https://doi.org/10.1016/j.jss.2018.09.063.

26. Londono JCG, Pelaez JAN, Salazar WAM, Madrid J, Restrepo MAM, Perez RAA, et al. Surgical site infection after breast cancer surgery at 30 days and associated factors. Infectio [Internet], 2016 [cited 2020 Feb 14]; 21(2):96-101. DOI: https://doi.org/10.1016/j.infect.2016.04.003.

27. Olsen MA, Ball KE, Katelin N, Wallace AE, Fraser VJ. Validation of ICD-9-CM Diagnosis Codes for Surgical Site Infection and Noninfectious Wound Complications After Mastectomy. Infect. Control. Hosp. Epidemiol. [Internet], 2017 [cited 2020 Jul 17]; 38(3):334-9. DOI: https://doi.org/10.1017/ice.2016.271.

28. Cruz LAP, Prado MAS, Ferreira SMA, Panobianco MS, Gozzo TO, Almeida AM. Occurrence of seroma post-mastectomy and care with aspiration drain in the Household. Rev. Enferm. UFPE on line. [Internet], 2017 [cited 2020 Jul 18]; 11(1):179-87. DOI: https://periodicos.ufpe.br/revistas/revistaenfermagem/article/view/11892.

29. Djordjevic M, BOJIC T, Djordjevic L, Budjevac D, Djordjevic N, Ignjatovic N, et al. Evaluation of Prognostic Factors Involved in Seroma Formation after Radical Surgery for Breast Cancer. Acta Fac. Med. Naiss. [Internet], 2018 [cited 2020 May 15]; 35(3):185-92. Available from: https://scindeks-clanci.ceon.rs/data/pdf/0351-6083/2018/0351-60831803185D.pdf.

30. Mendonça AF, Pimentel PP, Ribeiro SO, Mitiake SS, Irineu RJ. Anchor lipoabdominoplasty. Rev. bras. cir. plást. [Internet], 2019 [cited 2020 Mar 19]; 34(4):524-30. DOI: http://www.dx.doi.org/10.5935/2177-1235.2019RBCP0233.

31. Rodrigues DGOC. Complicações em abdominoplastia: experiência do serviço de cirurgia plástica do Hospital do Servidor Público Municipal de São Paulo [monografia de especialização]. São Paulo: Hospital do Servidor Público Municipal; 2017. [cited 2020 Mar 19]. Available from: http://docs.bvsalud.org/biblioref/2019/08/1008951/denis-guilherme-de-oliveira-colnagorodrigues.pdf.

32. Castrejón LLSL, Gavidia RCG. Obesidad como factor asociado a complicaciones en mastectomía [monografia de especialização]. Peru: Universidad Privada Antenor Orrego; 2019. [cited 2020 Mar 19]. Available from: http://repositorio.upao.edu.pe/handle/20.500.12759/5248.

33. D'Alessandro GS, Povedano A, Santos LKLL, Santos RA, Goes JCS. Immediate breast reconstruction with latissimus dorsi flap and silicone implant. Rev. Bras. Cir. Plást. [Internet], 2015 [cited 2020 Jun 14]; 30(2):163-71. DOI: https://doi.org/10.5935/21771235.2015RBCP0135.

34. Cammarota MC, Ribeiro I, Lima RQ, Almeida CM, Moura LG, Daher LMC, et al. The use of adhesion sutures to minimize the formation of seroma following mastectomy with immediate breast reconstruction. Rev Bras Cir Plást [Internet], 2016 [cited 2020 Jun 25]; 31(2):158-65. DOI: https://doi.org/10.5935/2177-1235.2016RBCP0026.

35. Nickel KB, Fox IK, Margenthaler JA, Wallace AE, Fraser VJ, Olsen MA. Effect of Noninfectious Wound Complications after Mastectomy on Subsequent Surgical Procedures and Early Implant Loss. J. Am. Coll. Surg. [Internet], 2016 [cited 2020 Aug 11 ]. 222(5):844-52. DOI: https://doi.org/10.1016/j.jamcollsurg.2016.01.050.

36. Momoh AO, Colakoglu S, Blacam C, Gautam S, Tobias AM, Lee BT. Delayed Autologous Breast Reconstruction After Postmastectomy Radiation Therapy: Is There an Optimal Time? Ann. Plast. Sur. [Internet], 2012 [cited 2020 Jul 25]; 69(1):14-8. DOI: https://doi.org/10.1097/SAP.0b013e31821ee4b6.

37. Sinha I, Pusic ALMD, Wilkins EGMD, Hamil JBM, Chen X, Kim HM, et al. Late Surgical-Site Infection in Immediate Implant-Based Breast Reconstruction. Plast. Reconstr. Surg. [Internet], 2017 [cited 2020 Mar 27]; 139(1):20-8. DOI: https://doi.org/10.1097/PRS.0000000000002839. 